\title{
HYRA gas-filled separator coupled to $4 \pi$ spin spectrometer at IUAC, New Delhi
}

\author{
N. Madhavan ${ }^{\text {la }}$, I. Mazumdar ${ }^{2}$, T. Varughese ${ }^{1}$, J. Gehlot ${ }^{1}$, S. Nath ${ }^{1}$, D. A. Gothe ${ }^{2}$, P. B. Chavan ${ }^{2}$, G. Mohanto ${ }^{1}$, \\ M. B. Naik ${ }^{3}$, I. Mukul ${ }^{1}$ and A. K. Sinha ${ }^{4}$ \\ ${ }^{1}$ Inter University Accelerator Centre, Aruna Asaf Ali Marg, New Delhi - 110067, India \\ ${ }^{2}$ Department of Nuclear and Atomic Physics, Tata Institute of Fundamental Research, Mumbai - 400005, India \\ ${ }^{3}$ Department of Physics, Karnatak University, Dharwad - 580003, India \\ ${ }^{4}$ UGC-DAE-CSR, Kolkata Centre, Kolkata - 700098, India
}

\begin{abstract}
The Hybrid Recoil mass Analyzer (HYRA) operated in gas-filled mode has recently been coupled with the TIFR $4 \pi$ spin spectrometer at IUAC, New Delhi to carry out spin (or angular momentum) distribution of heavy residues from fusion-evaporation reactions and spin gated GDR measurements. The details of the combined facility, initial experiments on evaporation residue tagged $\gamma$-multiplicity and the extraction of average transmission efficiency of HYRA are elaborated.
\end{abstract}

\section{Introduction}

The study of evaporation residue (ER) cross-section in heavy mass region probes the compound system in the pre-saddle region and can shed light on fusion-fission process, dissipation effects and optimum way to reach island of super heavy nuclei. The measurement of spin distribution of ERs offers additional constraints to critically test the various fusion-fission models as a single cross-section value at an excitation energy spans into a distribution of partial wave cross-sections. Any successful theoretical model for fusion-fission process should not only be able to reproduce the total ER crosssection but also the way it is distributed in various partial wave strengths. The spin distribution data available in heavy systems is sparse mainly due to the difficulty in achieving an efficient and clean ER tagging signal in the direction of primary beam. Recently, with such a study as one of the goals, the gas-filled part of HYRA [1] at IUAC, New Delhi has been coupled with the $4 \pi$ spin spectrometer [2] transported from TIFR, Mumbai. Few initial experiments have been carried out successfully using the coupled system.

The extraction of ER spin distribution beyond mass 180 amu have been carried out at IUAC [3 - 6] using Heavy Ion Reaction Analyzer (HIRA) [7] coupled with 14-element BGO multiplicity array in CN mass 190 - 205 amu region. The transmission efficiency of vacuum mode RMS, HIRA is too low in mass 200 amu region populated using asymmetric entrance channels possible with presently available beams at IUAC. This, combined

a e-mail: madhavan@iuac.res.in with the limited efficiency and granularity of BGO array, makes ER tagged $\gamma$-multiplicity measurements increasingly difficult. Hence, we decided to combine the spin spectrometer with gas-filled part of HYRA to extract the spin distribution in heavy mass region, through $\gamma$ multiplicity measurements, around ${ }^{200} \mathrm{~Pb} \mathrm{CN}$ and well beyond it.

The earlier study of high spin spectroscopy of ${ }^{254} \mathrm{No}$ at JYFL, Finland using RITU gas-filled separator coupled with few unsuppressed, germanium clover SARI detectors [8] and also at ANL, USA using FMA recoil mass spectrometer coupled with Gammasphere [9] have revealed the angular momentum up to which ${ }^{254} \mathrm{No}$ is populated (namely, about 20 ћ), by surviving fission. The higher ER detection efficiency of RITU in comparison with FMA resulted in similar or better statistics at high spin with a much lesser number of gamma detectors. Similarly, the coupling of a gas-filled separator of higher efficiency and selectivity with a spin spectrometer of higher efficiency and granularity is certainly a powerful and unique combination for spin distribution studies in heavy ERs, in the presence of overwhelming fission.

\section{HYRA - $4 \pi$ Spin spectrometer facility}

HYRA is a dual mode, dual stage spectrometer/separator funded by the Department of Science and Technology, Government of India and set up in the second beam hall downstream of superconducting LINAC booster [10] at 
IUAC. The first stage of electromagnetic configuration Q1Q2-MD1-Q3-MD2-Q4Q5 (where Q stands for magnetic quadrupole and MD for magnetic dipole) can be operated in gas-filled mode for heavy residue detection in normal kinematics. In vacuum mode, the same stage can be operated as a momentum achromat for secondary beam extraction from direct reactions or as a preseparator for second stage in inverse kinematics similar to ORNL RMS [11]. The second stage of configuration Q6Q7-ED-MD3-Q8Q9 (where ED stands for electrostatic dipole) is an energy dispersionless, mass spectrometer differing in design from ORNL RMS. Presently, the first stage of HYRA has been set up and is being used in gasfilled mode for heavy ER detection in the direction of primary beam.

The TIFR $4 \pi$ spin spectrometer facility consists of 32 conically-shaped sodium iodide crystals, 20 of which have hexagonal face and the remaining pentagonal face, in a closed packed geometry. The internal clearance is about $200 \mathrm{~mm}$. Each hexagonal detector has an absolute efficiency of $\sim 3 \%$ and each pentagonal detector is of $\sim 2 \%$ efficiency for a gamma ray of energy $660 \mathrm{keV}$. The whole array can be split into two halves to access the target chamber.

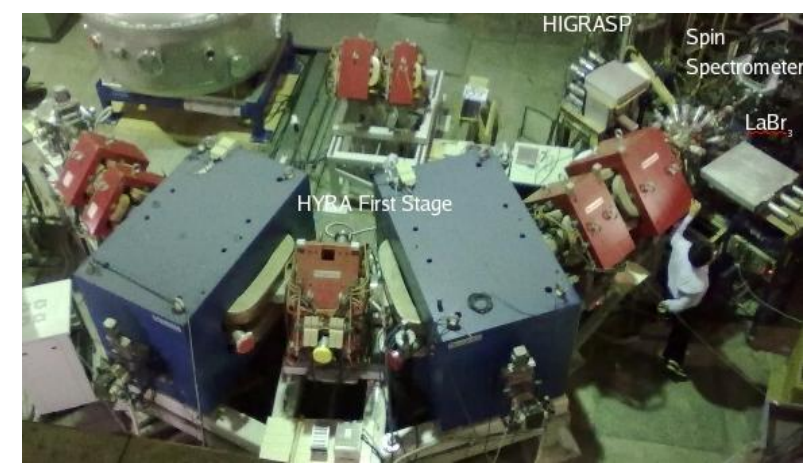

Fig. 1. HYRA - Spin spectrometer combined facility (beam enters from top right in the picture).

Special mounting and alignment arrangements were made to install the array at the target site of HYRA within the constraints allowed by the upstream clover mounting structure. The beam-line section upstream of the array was made telescopic to allow better access to the target chamber. Fig. 1 shows the HYRA - spin spectrometer combined facility with the beam entering from the top right corner. In most of the experiments 29 detectors were used with two pentagons giving way to beam entrance and exit. The third detector, a hexagonal one at $90^{\circ}$, was removed for target ladder operation or high energy gamma ray detection. The PMTs of the five forward most detectors were wrapped with $\mathrm{Mu}$-metal shield to overcome the gain reduction due to fringing field of Q1, which was operated up to $80 \%$ of maximum field in the experiments. In the first experiment, prior to the use of Mu-metal shields, signals from only 24 detectors were used. The threshold for energy signal from each detector was set at $\sim 100 \mathrm{keV}$. The energy signals were properly gain matched and the timing signals from all the detectors were matched. Two output signals, namely the sum energy and the timing "OR" signal of all detectors, were taken to data acquisition area to set up coincidence with HYRA focal plane signals.

\section{Experimental details and initial results}

Initial experiments carried out were fusion-evaporation reactions ${ }^{16} \mathrm{O}+{ }^{180} \mathrm{Hf} \rightarrow{ }^{196} \mathrm{Hg}^{*},{ }^{16} \mathrm{O}+{ }^{208} \mathrm{~Pb} \rightarrow{ }^{224} \mathrm{Th}^{*}$ and ${ }^{30} \mathrm{Si}+{ }^{170} \mathrm{Er} \rightarrow{ }^{200} \mathrm{~Pb}^{*}$ with the first one for studying ER and/or spin gated GDR decay and the latter two for studying the fission hindrance in heavy nuclei.

In all the above experiments, pulsed beam delivered by 15 UD Pelletron and superconducting LINAC (first module) combination were used. The pulse separation (either $2 \mu \mathrm{s}$ or $4 \mu \mathrm{s}$ ) was chosen as per the time-of-flight (TOF) of ERs through HYRA in order to check for background at the focal plane. The targets were mounted in a small pill-box type, aluminum chamber of $40 \mathrm{~mm}$ vertical clearance and $120 \mathrm{~mm}$ internal diameter (Fig. 2). Two monitor detectors are mounted, on either side at $24^{\circ}$ with respect to beam direction, for normalization. Counts in these and the current registered in the beam catcher in MD1 (with MD1 switched off) helps in proper beam tuning on the target, initially. Helium gas is fed through a port in the bottom lid upstream of target.

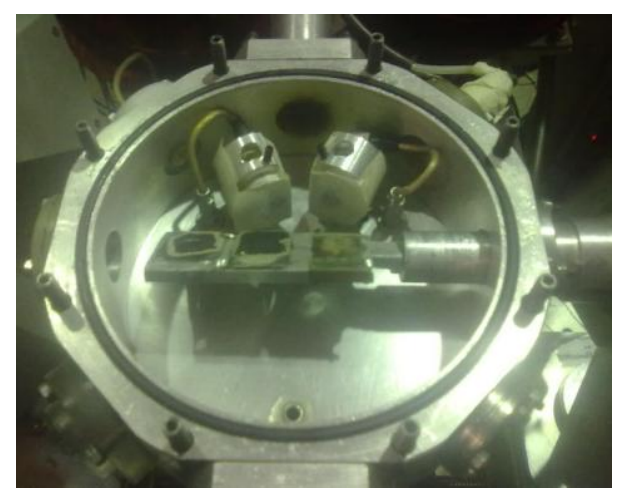

Fig. 2. Aluminum target chamber and accessories (beam enters from bottom, in the figure).

A thin carbon foil (thickness $\sim 150 \mu \mathrm{g} / \mathrm{cm}^{2}$ ), mounted $325 \mathrm{~mm}$ upstream of the target, served as the entrance window foil for gas-filled operation. Helium gas at a typical dynamical pressure of 0.15 Torr $(0.25$ Torr for higher energy ERs) was used in the separator and the pressure was exactly maintained using a MKS control unit and Baratron gauge-cum-solenoid valve combination.

The focal plane detection system consists of a 125 $\mathrm{mm} \times 50 \mathrm{~mm}$ window foil (to separate the helium and isobutane gas regions) of $0.5 \mu \mathrm{m}$ thick mylar followed by a $57 \mathrm{~mm}$ x $57 \mathrm{~mm}$ multi-wire proportional counter (MWPC) and a resistive $50 \mathrm{~mm}$ x $50 \mathrm{~mm}$ position sensitive silicon detector of $300 \mathrm{~mm}$ depletion depth. A 
movable stopper between the MWPC and silicon detectors helped in protecting the silicon detector during HYRA field optimization. The energy loss and position signals in MWPC, residual energy and position signals in silicon detector and a quantity related to TOF (measured by starting a Time to Amplitude Converter (TAC) with MWPC timing signal and stopping it by the beam RF signal) were used to distinguish between ERs and other background particles at the focal plane. Fig. 3 shows TAC spectra (for the ${ }^{16} \mathrm{O}+{ }^{180} \mathrm{Hf}$ fusion-evaporation reaction) started, in both cases, by MWPC timing signal and stopped by beam RF (upper panel) and stopped by suitably delayed "OR" signal from spin spectrometer (lower panel). Within the TAC range of $4 \mu \mathrm{s}$, mostly ERs are seen with only a small trace of scattered beam-like particles seen as a spike in upper panel. The ERs yield $\gamma$ folds (observed $\gamma$-multiplicity) up to 22 while the beamlike spike yields only up to 2 . The rejection factor of HYRA gas-filled separator for beam-like, target-like and fission fragments in this reaction is excellent.

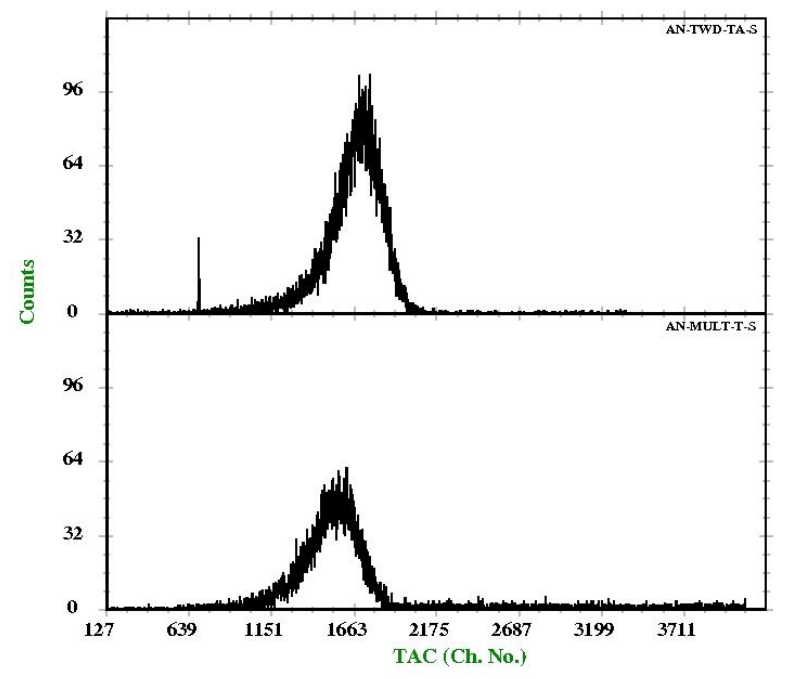

Fig. 3. TAC spectra started, in both cases, by MWPC timing signal and stopped by beam RF (top panel) and by delayed 'OR' signal of spin spectrometer (bottom panel). TAC range is $4 \mu \mathrm{s}$. Tiny spike to the left of ER peak in top panel is due to scattered beam-like particles.

${ }^{200} \mathrm{~Pb}^{*} \mathrm{CN}$ has been well studied through ER spin distribution using the fusion-evaporation reactions ${ }^{16} \mathrm{O}+$ ${ }^{184} \mathrm{~W}$ and ${ }^{19} \mathrm{~F}+{ }^{181} \mathrm{Ta}[4,5]$ at IUAC. As the ER crosssection has been measured for ${ }^{30} \mathrm{Si}+{ }^{170} \mathrm{Er}$ by Hinde et al. [12] leading to the same $\mathrm{CN}$, we chose to study the spin distribution for this reaction using the combined facility of HYRA and spin spectrometer to look for dissipation effects related to entrance channel mass asymmetry. Spin distribution has been measured for ERs selected by HYRA gas-filled mode in the excitation energy range of about 50 to $75 \mathrm{MeV}$ [13]. A clear reduction in mean gamma multiplicity of ERs for this system is seen, as the excitation energy increases, in comparison with the other two asymmetric systems. This has been explained in reference [13].

${ }^{16} \mathrm{O}+{ }^{208} \mathrm{~Pb} \rightarrow{ }^{224} \mathrm{Th}^{*}$ has already been studied through several probes such as evaporated neutrons, charged particles, ER cross section and GDR decay [14 17]. The additional information of ER spin distribution through $\gamma$-ray multiplicity has been attempted [18] in a limited range of excitation energies with the present combined facility. The large fission cross-section for this system results in a low ER cross-section and the measurement of associated spin distribution critically requires the large efficiency and cleaning power of HYRA gas-filled mode and the large efficiency and granularity of the $4 \pi$ spin spectrometer. This experiment may provide some idea on the lower extent of ER cross section for which the spin distribution can be effectively measured in a reasonable amount of time, which directly translates to the upper limit of the mass of $\mathrm{CN}$.

\section{Transmission efficiency of HYRA}

A common method used to extract the transmission efficiency of a spectrometer for the detection of a particular ER is to detect characteristic gamma rays from the ER in singles mode as well as with ER gating, using a high purity germanium detector. The ratio of the photopeak counts of an identified gamma ray in the ER gated spectrum to that in the singles spectrum gives the transmission efficiency of the spectrometer for that ER, with the gamma detector efficiency cancelling out. However, this is a time consuming method and needs extra detection system to be set up.

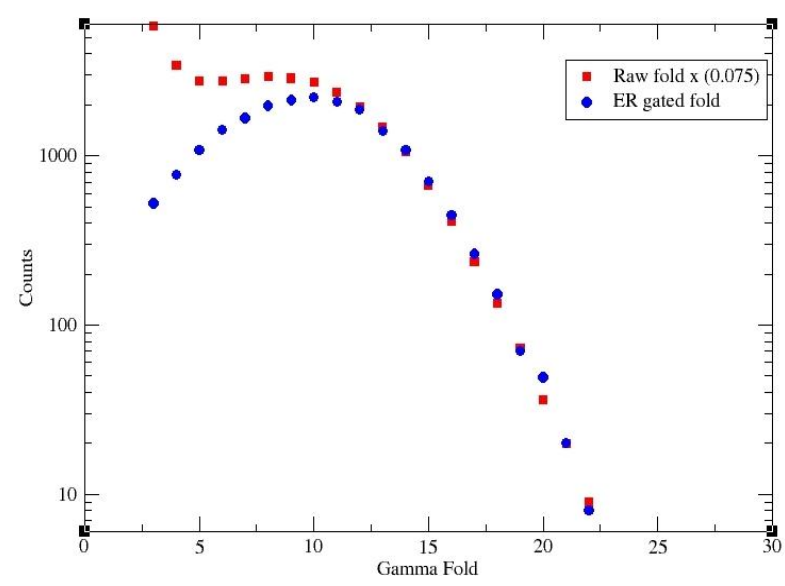

Fig. 4. ER gated $\gamma$-fold spectrum (filled circles) superimposed with singles $\gamma$-fold spectrum scaled down to $7.5 \%$ (filled squares) for the fusion-evaporation reaction ${ }^{16} \mathrm{O}+{ }^{180} \mathrm{Hf} \rightarrow$ ${ }^{196} \mathrm{Hg} *$ shows perfect matching of higher $\gamma$-folds indicating an average transmission efficiency of $7.5 \%$ for ERs detected at the focal plane of HYRA. 
We have adopted a modified method to extract the average transmission efficiency of HYRA gas-filled mode for all ERs detected at the focal plane using higher fold data in singles as well as ER gated $\gamma$-fold spectrum. The reason to consider only the higher $\gamma$-fold data is clear from Fig. 4 which shows the ER gated $\gamma$-fold spectrum superimposed with singles $\gamma$-fold spectrum (scaled down to $7.5 \%$ ) for ${ }^{16} \mathrm{O}+{ }^{180} \mathrm{Hf}$ reaction. As can be clearly seen from the departure from gated spectrum, the lower fold data in singles spectrum is heavily contaminated with gamma rays from (i) Coulomb excitation, (ii) fission fragments and (iii) fusion-evaporation reaction involving beam and carbon in entrance window foil. However, the higher fold data (fold 12 and above) perfectly match indicating an average transmission efficiency of $7.5 \%$ for all ERs detected at the focal plane.

However, it should be pointed out that this method may be employed if no other competing processes of larger angular momentum are produced in the reaction and/or detected at the focal plane along with ERs as in the present case.

\section{Summary and future plans}

The unique combination of gas-filled separator (HYRA) and TIFR $4 \pi$ spin spectrometer has recently been realized at IUAC, New Delhi to probe the limits of angular momentum in fusion-evaporation from heavy $\mathrm{CN}$. Initial experimental results are encouraging with negligible background at the focal plane. Comparison of three entrance channels leading to the same $\mathrm{CN}{ }^{200} \mathrm{~Pb}^{*}$, at similar excitation energies, reveal that for more symmetric system (studied with the facility elaborated here) the reduction in the normalized ER cross-section, at higher excitation energies, is accompanied by a reduction in average angular momentum. Though the symmetric combination brings in a lot more angular momentum in the entrance channel, effective angular momentum transferred to surviving ERs is smaller. A modified method comparing the higher $\gamma$-fold data of ER gated and singles spectra yields an average transmission efficiency of $7.5 \%$ for the detection of ERs using HYRA gas-filled mode in ${ }^{16} \mathrm{O}+{ }^{180} \mathrm{Hf} \rightarrow{ }^{196} \mathrm{Hg}^{*}$ fusion-evaporation reaction. We plan to increase the range of studies to higher masses and also to look at spin gated background events, when present, to pin-point the origin of such competing processes.

\section{Acknowledgments}

Funds for HYRA from DST, Govt. of India and efforts taken by TIFR for transportation and coupling of spin spectrometer with HYRA are acknowledged. Cooperation from other HYRA project investigators is appreciated. Dr. A. Roy, Dr. S. Kailas, Prof. S. S. Kapoor and Prof. G. K. Mehta for continuous support and encouragement, Dr. R. K. Bhowmik for his keen interest, A. Jhingan for focal plane detection system and E. Prasad for help are acknowledged.

\section{References}

1. N. Madhavan et al., Pramana Journal of Physics, 75, 317 (2010).

2. I. Mazumdar et al., DAE Symp. On Nucl. Phys., 53, 713 (2008).

3. S. K. Hui et al., Phys. Rev. C, 62, 054604 (2000).

4. P. D. Shidling et al., Phys. Lett. B. 670, 99 (2008).

5. P. D. Shidling et al., Phys. Rev. C. 74, 064603 (2006).

6. S. Nath et al., Nucl. Phys. A, 850, 22 (2011).

7. A. K. Sinha et al., Nucl. Instr. Meth. A 339, 543 (1994).

8. M. Leino et al., Eur. Phys. J. A 6, 63 (1999)

9. P. Reiter et al. Phys. Rev. Lett., 82, 509 (1999).

10. P. N. Prakash et al., Pramana Journal of Physics, 59, 849 (2002).

11. J.D. Cole, et al., Nucl. Instr. and Meth. B 70, 343 (1992).

12. D. J. Hinde, et al., Nucl. Phys. A 385, 109 (1982).

13. G. Mohanto et al., Proceedings of this conference (FUSION11), submitted.

14. J.P. Lestone et al., Phys. Rev. Lett. 67, 1078 (1991).

15. D.J. Hinde et al., Phys. Rev. C 45, 1229 (1992).

16. B.B. Back et al., Phys. Rev. C 60, 044602 (1999).

17. I. Dioszegi et al., Phys. Rev. C 61, 024613 (2000).

18. M. B. Naik et al., DAE symposium on Nuclear Physics, Vol. 55, 312 (2010). 